Faculty of Mathematical Sciences

\section{University of Twente}

University for Technical and Social Sciences
P.O. Box 217

7500 AE Enschede

The Netherlands

Phone: +31-53-4893400

Fax: +31-53-4893114

Email: memo@math.utwente.nl

Memorandum No. 1471

On stability robustness with respect to

LTV uncertainties

G. Meinsma, T. Inasaki ${ }^{1}$ and M. Fu ${ }^{2}$

November 1998

ISSN 0169-2690

\footnotetext{
${ }^{1}$ Dept. of Control Systems Engineering, Tokyo Institute of Technology, 2-12-1 Oookayama, Meguro, Tokyo 152, Japan

${ }^{2}$ Dept. of Electrical and Computer Engineering, University of Newcastle, Callaghan, NSW 2308, Australia
} 


\title{
On stability robustness with respect to LTV uncertainties
}

\author{
Gjerrit Meinsma \\ Department of Systems, Signals and Control \\ Faculty of Mathematical Sciences \\ University of Twente \\ P.O. Box 217, 7500 AE Enschede \\ The Netherlands \\ g.meinsma@math. utwente.nl \\ Tetsuya Iwasaki \\ Department of Control Systems Engineering \\ Tokyo Institute of Technology \\ 2-12-1 Oookayama, Meguro, Tokyo 152 \\ Japan \\ iwasaki@ctrl.titech.ac.jp \\ Minyue Fu \\ Department of Electrical and Computer Engineering \\ University of Newcastle \\ Callaghan, NSW 2308 \\ Australia. \\ eemflee.newcastle.edu.au
}

\begin{abstract}
It is shown that the well-known $(D, G)$-scaling upper bound of the structured singular value is a nonconservative test for robust stability with respect to certain linear time-varying uncertainties.
\end{abstract}

\section{Keywords}

Mixed structured singular values, Duality, Linear matrix inequalities, Time-varying systems, Robustness, IQC.

1991 Mathematics Subject Classification: 93B36, 93C50, 93D09, 93D25. 


\section{Introduction}

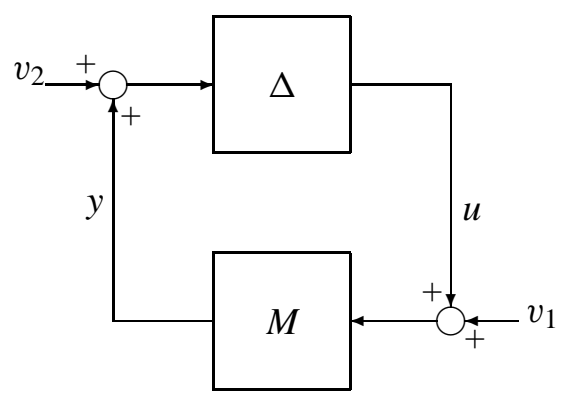

Figure 1: The closed loop.

Is the above closed loop stable for all $\Delta$ 's in a given set of stable operators $\mathcal{B}$ ? That, roughly, is the fundamental robust stability problem.

There is an intriguing result by Megretski and Treil [4] and Shamma [8] which says, loosely speaking, that if $M$ is a stable LTI operator and the set of $\Delta$ 's is the set of contractive linear time-varying operators of some fixed block diagonal structure

$$
\Delta=\operatorname{diag}\left(\Delta_{1}, \Delta_{2}, \ldots, \Delta_{m_{F}}\right),
$$

that then the closed loop is robustly stable - that is, stable for all such $\Delta$ 's—if and only if the $\mathcal{H}_{\infty}$-norm of $D M D^{-1}$ is less than one for some constant diagonal matrix $D$ that commutes with the $\Delta$ 's. The problem can be decided in polynomial time, and it is a problem that has since long been associated with an upper bound of the structured singular value. The intriguing part is that the result holds for any number of LTV blocks $\Delta_{i}$, which is in stark contrast with the case that the $\Delta_{i}$ 's are assumed time-invariant.

Paganini [6] extended this result by allowing for the more general block diagonal structure

$$
\Delta=\operatorname{diag}\left(\delta_{1} I_{n_{1}}, \ldots, \delta_{m_{c}} I_{n_{m_{c}}}, \Delta_{1}, \ldots, \Delta_{m_{F}}\right) .
$$

A precise definition is given in Section 2. Paganini's result is an exact generalization and leads, again, to a convex optimization problem over the constant matrices $D$ that commute with $\Delta$.

In view of the connection of these results with the upper bounds of the structured singular it is natural to ask if the well known $(D, G)$-scaling upper bound of the mixed structured singular value also has a similar interpretation. In this note we show that that is indeed the case.

The $(D, G)$-scaling upper bound of the structured singular value was originally defined as a means to provide an easy-to-verify condition that guarantees robust stability with respect to the contractive linear time-invariant operators $\Delta$ of the form

$$
\Delta=\operatorname{diag}\left(\tilde{\delta}_{1} I_{\tilde{n}_{1}}, \ldots, \tilde{\delta}_{m_{r}} I_{\tilde{n}_{m_{r}}}, \delta_{1} I_{n_{1}}, \ldots, \delta_{m_{c}} I_{n_{m_{c}}}, \Delta_{1}, \ldots, \Delta_{m_{F}}\right),
$$

with $\tilde{\delta}_{i}$ denoting real-valued constants [1]. It is known that for general LTI plants $M$ this sufficient condition is necessary as well if and only if,

$$
2\left(m_{r}+m_{c}\right)+m_{F} \leq 3 .
$$


(See [5].) In this note we show that the $(D, G)$-scaling condition is in fact both necessary and sufficient for robust stability with respect to the contractive LTV operators $\Delta$ of the form (3) with now $\tilde{\delta}_{i}$ denoting linear time-varying self-adjoint operators on $\ell_{2}$. A precise definition follows. Paganini [7] has gone through considerable trouble to show that for his structure (2) one may assume causality of $\Delta$ without changing the condition. In the extended structure (3) with self-adjoint $\tilde{\delta}_{i}$ this is no longer possible.

\section{Notation and preliminaries}

$\ell_{2}:=\left\{x: \mathbb{Z} \mapsto \mathbb{R}: \sum_{k \in \mathbb{Z}} x^{2}(k)<\infty\right\}$. The norm $\|v\|_{2}$ of $v \in \ell_{2}$ is the usual norm on $\ell_{2}$ and for vector-valued signals $v \in \ell_{2}^{n}$ the norm $\|v\|_{2}$ is defined as $\left(\left\|v_{1}\right\|_{2}^{2}+\cdots+\right.$ $\left.\left\|v_{n}\right\|_{2}^{2}\right)^{1 / 2}$. The induced norm is denoted by $\|\cdot\|$. So, for $F: \ell_{2}^{n} \mapsto \ell_{2}^{n}$ it is defined as $\|F\|:=\sup _{u \in \ell_{2}^{n}}\|F u\|_{2} /\|u\|_{2}$. For matrices $F \in \mathbb{C}^{n \times m}$ the induced norm will be the spectral norm, and for vectors this reduces to the Euclidean norm.

$F^{\mathrm{H}}$ is the complex conjugate transpose of $F$, and $\mathrm{He} F$ is the Hermitian part $F$ defined as $\mathrm{He} F=\frac{1}{2}\left(F+F^{\mathrm{H}}\right)$.

An operator $\Delta: \ell_{2}^{n} \mapsto \ell_{2}^{n}$ is said to be contractive if $\|\Delta v\|_{2} \leq\|v\|_{2}$ for every $v \in \ell_{2}^{n}$. Lower case $\delta$ 's always denote operators from $\ell_{2}^{1}$ to $\ell_{2}^{1}$. Then for $u, y \in \ell_{2}^{n}$ the expression $y=\delta I_{n} u$ is defined to mean that the entries $y_{k}$ of $y$ satisfy $y_{k}=\delta u_{k}$. An operator $\delta: \ell_{2} \mapsto \ell_{2}$ is self-adjoint if $\langle u, \delta v\rangle=\langle\delta u, v\rangle$ for all $u, v \in \ell_{2}$.

The $M$ and $\Delta$ throughout denote bounded operators from $\ell_{2}^{n}$ to $\ell_{2}^{n}$ and $M$ is assumed linear time invariant (LTI). Bounded operators on $\ell_{2}^{n}$ are also called stable.

Hats will denote $Z$-transforms, so if $y \in \ell_{2}$ then $\hat{y}(z)$ is defined as $\hat{y}(z)=\sum_{k \in \mathbb{Z}} y(k) z^{-k}$. To avoid clutter we shall use for functions $\hat{f}$ of frequency the notation

$$
\hat{f}_{\omega}:=\hat{f}\left(e^{i \omega}\right)
$$

\subsection{Stability}

The closed loop depicted in Fig. 1 is considered internally stable if the map from $\left[\begin{array}{l}v_{1} \\ v_{2}\end{array}\right]$ to $\left[\begin{array}{l}u \\ y\end{array}\right]$ is bounded as a map from $l_{2}^{2 n}$ to $l_{2}^{2 n}$. Because of stability of $M$ and $\Delta$ the closed loop is internally stable iff $(I-\Delta M)^{-1}$ is bounded. The closed loop will be called uniformly robustly stable with respect to some set $\mathcal{B}$ of stable LTV operators if there is an $\gamma>0$ such that

$$
\left\|\left[\begin{array}{l}
u \\
y
\end{array}\right]\right\|_{2} \leq \gamma\left\|\left[\begin{array}{l}
v_{1} \\
v_{2}
\end{array}\right]\right\|_{2} \quad \forall \Delta \in \mathcal{B},\left[\begin{array}{l}
v_{1} \\
v_{2}
\end{array}\right] \in \ell_{2}^{2 n}
$$

We only consider $\Delta$ 's with norm at most one and stable $M$. In that case (4) holds if and only if there is an $\epsilon>0$ such that

$$
\|(I-\Delta M) u\|_{2} \geq \epsilon\|u\|_{2} \quad \forall \Delta \in \mathcal{B}, u \in \ell_{2}^{n} .
$$

\subsection{The $\Delta$ 's and the $(D, G)$-scaling matrices}

Throughout we assume that $\Delta: \ell_{2}^{n} \mapsto \ell_{2}^{n}$ and that $\Delta$ is of the form

$$
\Delta=\operatorname{diag}\left(\tilde{\delta}_{1} I_{\tilde{n}_{1}}, \ldots, \tilde{\delta}_{m_{r}} I_{\tilde{n}_{m_{r}}}, \delta_{1} I_{n_{1}}, \ldots, \delta_{m_{c}} I_{n_{m_{c}}}, \Delta_{1}, \ldots, \Delta_{m_{F}}\right)
$$


with

$$
\left\{\begin{array}{rlll}
\tilde{\delta}_{i} & : & \ell_{2} \mapsto \ell_{2} & \text { LTV, self-adjoint and }\left\|\tilde{\delta}_{i}\right\| \leq 1, \\
\delta_{i}: & \ell_{2} \mapsto \ell_{2} & \text { LTV and }\left\|\delta_{i}\right\| \leq 1, \\
\Delta_{i} & : & \ell_{2}^{q_{i}} \mapsto \ell_{2}^{q_{i}} & \text { LTV and }\left\|\Delta_{i}\right\| \leq 1 .
\end{array}\right.
$$

The dimensions and numbers $\tilde{n}_{i}, n_{i}, q_{i}, m_{r}, m_{c}, m_{F}$ of the various identity matrices and $\Delta_{i}$ blocks are fixed, but otherwise $\Delta$ may vary over all possible $n \times n$ LTV operators of the form (5), (6). Given that, the sets $\mathcal{D}$ and $\mathcal{G}$ of $D$ and $G$-scales are defined accordingly as

$$
\begin{aligned}
& \mathcal{D}=\left\{D=\operatorname{diag}\left(\tilde{D}_{1}, \ldots, \tilde{D}_{m_{r}}, D_{1}, \ldots, D_{m_{c}}, d_{1} I_{q_{1}}, \ldots, d_{m_{F}} I_{q_{m_{F}}}\right)\right. \\
& \left.: 0<D=D^{\mathrm{T}} \in \mathbb{R}^{n \times n}\right\} \text {, } \\
& \mathcal{G}=\left\{G=\operatorname{diag}\left(\tilde{G}_{1}, \ldots, \tilde{G}_{m_{r}}, 0, \ldots, 0,0, \ldots, 0\right)\right. \\
& \left.: G=G^{\mathrm{H}} \in j \mathbb{R}^{n \times n}\right\} .
\end{aligned}
$$

Note that the $D$-scales are assumed real-valued and that the $G$-scales are taken to be purely imaginary. As it turns out there is no need to consider a wider class of $D$ and $G$-scales.

\section{The discrete-time result}

Theorem 3.1. The discrete-time closed-loop in Fig. 1 with stable LTI plant with transfer matrix $M$ is uniformly robustly stable with respect to $\Delta$ 's of the form $(5,6)$ if and only if there is a constant matrix $D \in \mathcal{D}$ and a constant matrix $G \in \mathcal{G}$ such that

$$
M_{\omega}^{\mathrm{H}} D M_{\omega}+j\left(G M_{\omega}-M_{\omega}^{\mathrm{H}} G\right)-D<0 \quad \forall \omega \in[0,2 \pi] .
$$

The existence of such $D$ and $G$ can be tested in polynomial time. The remainder of this paper is devoted to a proof of this result. Megretski [3] showed this for the full blocks case (1), Paganini [6] derived this result for the case that the $\Delta$ 's are of the form (2). The proof of the general case (5) follows the same lines as that of [6] and [5]. A key idea is to replace the condition of the contractive $\Delta$-blocks with an integral quadratic condition independent of $\Delta$ :

Lemma 3.2. Let $u, y \in \ell_{2}^{q}$ and consider the quadratic integral

$$
\Sigma(u, y):=\int_{0}^{2 \pi}\left(\hat{y}_{\omega}-\hat{u}_{\omega}\right)\left(\hat{y}_{\omega}+\hat{u}_{\omega}\right)^{\mathrm{H}} d \omega \in \mathbb{R}^{q \times q} .
$$

The following holds.

1. There is a contractive self-adjoint LTV $\tilde{\delta}: \ell_{2} \mapsto \ell_{2}$ such that $u=\tilde{\delta} I_{q} y$ if and only if $\Sigma(u, y)$ is Hermitian and nonnegative definite.

2. There is a contractive LTV $\delta: \ell_{2} \mapsto \ell_{2}$ such that $u=\delta I_{q} y$ if and only if the Hermitian part of $\Sigma(u, y)$ is nonnegative definite.

3. There is a contractive LTV $\Delta: \ell_{2}^{q} \mapsto \ell_{2}^{q}$ such that $u=\Delta y$ if and only if the trace of $\Sigma(u, y)$ is nonnegative. 
Proof. See appendix.

A consequence of this result is the following.

Lemma 3.3. Let $u$ be a nonzero element of $\ell_{2}^{n}$. Then $(I-\Delta M) u=0$ for some $\Delta$ of the form $(5,6)$ if-and-only-if

$$
\Sigma(u, M u):=\int_{0}^{2 \pi}\left(M_{\omega}-I\right) \hat{u}_{\omega} \hat{u}_{\omega}^{\mathrm{H}}\left(M_{\omega}+I\right)^{\mathrm{H}} d \omega
$$

is of the form

$\left[\begin{array}{ccc|ccc|ccc}\tilde{Z}_{1} & ? & ? & ? & ? & ? & ? & ? & ? \\ ? & \tilde{Z}_{2} & ? & ? & ? & ? & ? & ? & ? \\ ? & ? & \ddots & ? & ? & ? & ? & ? & ? \\ \hline ? & ? & ? & \bar{Z}_{1} & ? & ? & ? & ? & ? \\ ? & ? & ? & ? & \bar{Z}_{2} & ? & ? & ? & ? \\ ? & ? & ? & ? & ? & \ddots & ? & ? & ? \\ \hline ? & ? & ? & ? & ? & ? & Z_{1} & ? & ? \\ ? & ? & ? & ? & ? & ? & ? & Z_{2} & ? \\ ? & ? & ? & ? & ? & ? & ? & ? & \ddots\end{array}\right] \in \mathbb{R}^{n \times n}$,

with $\tilde{Z}_{i}=\tilde{Z}_{i}^{\mathrm{T}} \geq 0, \mathrm{He} \bar{Z}_{i} \geq 0, \operatorname{Tr} Z_{i} \geq 0$, and with "?" denoting an irrelevant entry. Here the partitioning of (10) is compatible with that of $\Delta$.

Proof (sketch). The equation $(I-\Delta M) u=0$ is the same as

$$
u=\Delta M u \text {. }
$$

With appropriate partitionings, the expression $u=\Delta M u$ can be written row-block by rowblock as

$$
\begin{aligned}
u_{1} & =\tilde{\delta}_{1} M_{1} u \\
u_{2} & =\tilde{\delta}_{2} M_{2} u \\
\vdots & \vdots \\
u_{K} & =\Delta_{m_{F}} M_{K} u .
\end{aligned}
$$

By Lemma 3.2 there exist contractive $\tilde{\delta}_{i}, \delta_{i}$ and $\Delta_{i}$ of the form (6) for which the above equalities hold iff certain quadratic integrals $\Sigma_{i}$ have certain properties. It is not to difficult to figure out that these quadratic integrals $\Sigma_{i}$ are exactly the blocks on the diagonal of $\Sigma(u, M u)$, and that the conditions on these blocks are that they satisfy $\Sigma_{i}=\Sigma_{i}^{\mathrm{T}} \geq 0$, He $\Sigma_{i} \geq 0$, or $\operatorname{Tr} \Sigma_{i} \geq 0$, corresponding to the three types of uncertainties.

Proof of Theorem 3.1. Suppose such $D \in \mathcal{D}$ and $G \in \mathcal{G}$ exist. Then a standard argument will show that there is an $\epsilon>0$ such that $\|(I-\Delta M) u\|_{2} \geq \epsilon\|u\|_{2}$ for all $u$ and contractive $\Delta$ of the form (5). This is the definition of uniformly robustly stable. 
Conversely suppose the closed loop is uniformly robustly stable. For some $\epsilon>0$, then, $\|(I-\Delta M) u\|_{2} \geq \epsilon$ for every $u$ of unit norm. Define

$$
\mathcal{W}:=\left\{\Sigma(u, M u):\|u\|_{2}=1\right\} \subset \mathbb{R}^{n \times n} .
$$

By application of Lemma 3.3, the set $\mathcal{W}$ does not intersect the convex cone $\mathcal{Z}$ defined as

$$
Z:=\left\{Z: Z \text { is of the form (10) with } \tilde{Z}_{i}=\tilde{Z}_{i}^{\mathrm{T}} \geq 0, \operatorname{He} \bar{Z}_{i} \geq 0, \operatorname{Tr} Z_{i} \geq 0\right\} .
$$

In the appendix we show that in fact $\mathcal{W}$ is bounded away from $Z$. Remarkably the closure $\overline{\mathcal{W}}$ of $\mathcal{W}$ is convex. This observation is from Megretski \& Treil [4], and for completeness a proof is listed in the appendix, Lemma 5.1. Because $\mathcal{W}$ is bounded away from $\mathcal{Z}$, also the closure $\overline{\mathcal{W}}$ is bounded away from $Z$, so there is a $\gamma>0$ such that $\overline{\mathcal{W}}$ also does not intersect

$$
z_{\gamma}:=Z+\left\{Z \in \mathbb{R}^{n \times n}:\|Z\| \leq \gamma\right\} .
$$

Both $\overline{\mathcal{W}}$ and $Z_{\gamma}$ are convex and have empty intersection, and therefore a hyper-plane exists that separates the two sets [2, p.133]. In other words there is a nonzero matrix $E \in \mathbb{R}^{n \times n}$ (say of unit norm) such that ${ }^{1}$

$$
\langle E, \overline{\mathcal{W}}\rangle \leq\left\langle E, z_{\gamma}\right\rangle
$$

As inner product take $\langle X, Y\rangle=\operatorname{Tr} X^{\mathrm{T}} Y$. In particular (12) says that $\langle E, Z\rangle$ is bounded from below. By Lemma 5.3 that is the case if and only if $E$ is of the form

$$
E=\operatorname{diag}\left(\tilde{E}_{1}, \ldots, \tilde{E}_{m_{r}}, E_{1}, \ldots, E_{m_{c}}, e_{1} I, \ldots, e_{m_{F}} I\right)
$$

with $\tilde{E}_{i}+\tilde{E}_{i}^{\mathrm{T}} \geq 0, E_{i}=E_{i}^{\mathrm{T}} \geq 0$ and $0 \leq e_{i} \in \mathbb{R}$, that is, if and only if $E \in \overline{\mathcal{D}+j \mathcal{G}}$. In that case $\inf \langle E, Z\rangle=0$, and so

$$
a_{\gamma}:=\inf \left\langle E, Z_{\gamma}\right\rangle<0 \text {. }
$$

From (12) we thus see that $\langle E, \overline{\mathcal{W}}\rangle \leq a_{\gamma}<0$. If $\|u\|_{2}=1$, then

$$
\begin{aligned}
\int_{0}^{2 \pi} \hat{u}_{\omega}^{\mathrm{H}} & \left.\operatorname{He}\left(M_{\omega}+I\right)^{\mathrm{H}} E\left(M_{\omega}-I\right)\right) \hat{u}_{\omega} d \omega \\
= & \operatorname{Re} \operatorname{Tr} \int_{0}^{2 \pi} E\left(M_{\omega}-I\right) \hat{u}_{\omega} \hat{u}_{\omega}^{\mathrm{H}}\left(M_{\omega}+I\right)^{\mathrm{H}} d \omega \\
= & \langle E, \Sigma(u, M u)\rangle \leq \sup \langle E, \mathcal{W}\rangle \leq a_{\gamma}<0 .
\end{aligned}
$$

This being at most $a_{\gamma}<0$ for every $u \in \ell_{2}^{n},\|u\|_{2}=1$ implies that

$$
\operatorname{He}\left(M_{\omega}+I\right)^{\mathrm{H}}(E+\epsilon I)\left(M_{\omega}-I\right)<0 \quad \forall \omega \in[0,2 \pi],
$$

for some small enough $\epsilon>0$. Express $E+\epsilon I$ as $E+\epsilon I=D+j G$ for some $D \in \mathcal{D}$ and $G \in \mathcal{G}$. Then Equation (14) becomes (7).

\footnotetext{
${ }^{1}$ In (12) the expression $\langle E, \overline{\mathcal{W}}\rangle$ denotes the set $\{x: x=\langle E, Y\rangle, Y \in \overline{\mathcal{W}}\}$ and the inequality in (12) is defined to mean that every element of the set on the left-hand side, $\langle E, \overline{\mathcal{W}}\rangle$, is less than or equal to every element of the set on the right-hand side, $\left\langle E, Z_{\gamma}\right\rangle$.
} 


\section{The continuous-time result}

Analagous to the discrete-time case we say that a continuous-time system is uniformly robustly stable if there is a $\gamma>0$ such that (4) holds for all $v_{1}, v_{2} \in L_{2}$. Completely analagous to the discrete-time case it can be shown that:

Theorem 4.1. The continuous-time closed-loop in Fig. 1 with stable LTI plant with transfer matrix $M$ is uniformly robustly stable with respect to $\Delta$ 's of the form (5) with

$$
\left\{\begin{array}{rlll}
\tilde{\delta}_{i}: & L_{2} \mapsto L_{2} & \text { LTV, self-adjoint and }\left\|\tilde{\delta}_{i}\right\| \leq 1, \\
\delta_{i}: & L_{2} \mapsto L_{2} & \text { LTV and }\left\|\delta_{i}\right\| \leq 1, \\
\Delta_{i}: & L_{2}^{q_{i}} \mapsto L_{2}^{q_{i}} & \text { LTV and }\left\|\Delta_{i}\right\| \leq 1 .
\end{array}\right.
$$

if and only if there is a constant matrix $D \in \mathcal{D}$ and a constant matrix $G \in \mathcal{G}$ such that

$$
M(j \omega)^{\mathrm{H}} D M(j \omega)+j\left(G M(j \omega)-M(j \omega)^{\mathrm{H}} G\right)-D<0
$$

for all $\omega \in \mathbb{R} \cup \infty$.

\section{Appendix}

Proof of Lemma 3.2. Items 2 and 3 are proved in [6] (note that the Hermitian part of (8) is $\int_{0}^{2 \pi} \hat{y}_{\omega} \hat{y}_{\omega}^{\mathrm{H}}-\hat{u}_{\omega} \hat{u}_{\omega}^{\mathrm{H}} d \omega$, and its trace equals $\left.2 \pi\left(\|y\|_{2}^{2}-\|u\|_{2}^{2}\right)\right)$.

If $u:=\tilde{\delta} I_{q} y$ with $\tilde{\delta}$ self-adjoint and contractive then (8) is easily seen to be Hermitian and $\geq 0$. Conversely suppose ( 8 ) is Hermitian and nonnegative. Now let $\left\{f_{i}\right\}_{i=0,1,2, \ldots}$ be an orthonormal basis of $\ell_{2}$, and expand $y \in \ell_{2}^{q}$ in this basis:

$$
y=\sum_{j=0,1, \ldots} \gamma(j) f_{j}, \quad \gamma(j) \in \mathbb{R}^{q} .
$$

We may associate with this expansion the matrix $Y \in \mathbb{R}^{\infty \times q}$ of coefficients

$$
Y=\left[\begin{array}{cccc}
\gamma_{1}(0) & \gamma_{2}(0) & \cdots & \gamma_{q}(0) \\
\gamma_{1}(1) & \gamma_{2}(1) & \cdots & \gamma_{q}(1) \\
\gamma_{1}(2) & \gamma_{2}(2) & \cdots & \gamma_{q}(2) \\
\vdots & \vdots & \vdots & \vdots
\end{array}\right]
$$

The matrix $U$ is likewise defined from $u$. In this matrix notation the expression $u=\tilde{\delta} I_{q} y$ becomes $U=\tilde{\Delta} Y$, and the quadratic integral (8) becomes

$$
\Sigma(u, y)=\left(Y^{\mathrm{T}}-U^{\mathrm{T}}\right)(Y+U) .
$$

By assumption the above is Hermitian and nonnegative definite, that is,

$$
Y^{\mathrm{T}} U=U^{\mathrm{T}} Y \quad \text { and } \quad U^{\mathrm{T}} U \leq Y^{\mathrm{T}} Y .
$$

We may assume without loss of generality that the orthonormal basis $\left\{f_{j}\right\}$ was chosen such that the first, say $p$, elements $\left\{f_{1}, \ldots, f_{p}\right\}$ span the space spanned by the entries $\left\{y_{1}, \ldots, y_{q}\right\}$ of $y$. Then $Y$ is of the form

$$
Y=\left[\begin{array}{c}
I_{p} \\
0_{\infty \times p}
\end{array}\right] C \text { for some full row rank } C \in \mathbb{R}^{p \times q} .
$$


Then the second inequality of (15) is that $U^{\mathrm{T}} U \leq C^{\mathrm{T}} C$. This implies that $U$ is of the form $U=V C$ for some $V$. Partition $V$ as $\left[\begin{array}{l}V_{1} \\ V_{2}\end{array}\right]$ with $V_{1} \in \mathbb{R}^{p \times p}$. The two formulas of (15) then become

$$
C^{\mathrm{T}} V_{1} C=C^{\mathrm{T}} V_{1}^{\mathrm{T}} C \quad \text { and } \quad C^{\mathrm{T}}\left(V_{1}^{\mathrm{T}} V_{1}+V_{2}^{\mathrm{T}} V_{2}\right) C \leq C^{\mathrm{T}} I_{p} C .
$$

As $C$ has full row rank, (16) is equivalent to that

$$
V_{1}=V_{1}^{\mathrm{T}} \quad \text { and } \quad V_{1}^{\mathrm{T}} V_{1}+V_{2}^{\mathrm{T}} V_{2} \leq I_{p} .
$$

It is now immediate that $U$ equals $U=\tilde{\Delta} Y$ for $\tilde{\Delta}$ defined as

$$
\tilde{\Delta}:=\left[\begin{array}{cc}
V_{1} & V_{2}^{\mathrm{T}} \\
V_{2} & -V_{2} V_{1}\left(I-V_{1}^{2}\right)^{-1} V_{2}^{\mathrm{T}}
\end{array}\right] .
$$

It is easy to verify that $\tilde{\Delta}$ is contractive. Furthermore $\tilde{\Delta}$ is symmetric and so the corresponding operator $\tilde{\delta}$ is self-adjoint.

(It may happen that $I-V_{1}^{2}$ is singular. In that case the inverse in (17) may be replaced with the Moore-Penrose inverse.)

Lemma 5.1. The closure of (11) is convex.

Proof. The proof hinges on the fact that $\lim _{N \rightarrow \infty}\left\langle u, \sigma^{N} v\right\rangle=0$ for every pair $u, v \in \ell_{2}^{n}$ and with $\sigma^{N}$ denoting the $N$-step delay.

Let $u, v \in \ell_{2}^{n}$ both have unit norm, i.e., $\Sigma(u, M u), \Sigma(v, M v) \in \mathcal{W}$. Given $N \in \mathbb{N}$ and $\lambda \in[0,1]$ define $x$ as

$$
x:=\sqrt{\lambda} u+\sqrt{1-\lambda} \sigma^{N} v .
$$

Since $\Sigma$ is linear in its two arguments, we have that

$$
\begin{aligned}
\Sigma(x, M x)= & \lambda \Sigma(u, M u)+\sqrt{1-\lambda} \sqrt{\lambda} \Sigma\left(u, M \sigma^{N} v\right) \\
& +\sqrt{1-\lambda} \sqrt{\lambda} \Sigma\left(\sigma^{N} v, M u\right)+(1-\lambda) \Sigma(v, M v) .
\end{aligned}
$$

As $N \rightarrow \infty$ the contributions of $\Sigma\left(u, M \sigma^{N} v\right)$ and $\Sigma\left(\sigma^{N} v, M u\right)$ tend to zero, so

$$
\lim _{N \rightarrow \infty} \Sigma(x, M x)=\lambda \Sigma(u, M u)+(1-\lambda) \Sigma(v, M v) .
$$

That this is an element of the closure of (11) follows from the fact that. $\lim _{N \rightarrow \infty}\|x\|_{2}^{2}=$ $\lambda\|u\|_{2}^{2}+(1-\lambda)\|v\|_{2}^{2}=1$.

Lemma 5.2. Uniform robust stability implies that $\mathcal{W}$ is bounded away from $Z$.

Proof. Suppose to the contrary that

$$
\inf _{u \in \ell_{2}^{n},\|u\|_{2}=1, Z \in Z}\|\Sigma(u, M u)-Z\|=0 .
$$

This means that there is a sequence $\left\{u^{k}, Q_{k}\right\}_{k \in \mathbb{N}} \subset \ell_{2}^{n} \times \mathbb{R}^{n \times n}$ such that

$$
\Sigma\left(u^{k}, M u^{k}\right)+Q_{k} \in Z, \quad\left\|u^{k}\right\|_{2}=1, \quad \lim _{k \rightarrow \infty}\left\|Q_{k}\right\|=0 .
$$


For each $k$ define $y^{k}:=M u^{k} \in \ell_{2}^{n}$ and take $z^{k}$ to be any element of $\ell_{2}^{n}$ whose entries are mutually orthogonal and have unit norm, $\left\langle z_{i}^{k}, z_{j}^{k}\right\rangle=\delta_{i j}$, and whose entries are also orthogonal to all entries of $u^{k}$ and $y^{k}$. With it define

$$
\begin{aligned}
& \bar{u}^{k}:=u^{k}+\frac{1}{2}\left(\sqrt{\left\|Q_{k}\right\|} I_{n}-\frac{1}{\sqrt{\left\|Q_{k}\right\|}} Q_{k}\right) z^{k}, \\
& \bar{y}^{k}:=y^{k}+\frac{1}{2}\left(\sqrt{\left\|Q_{k}\right\|} I_{n}+\frac{1}{\sqrt{\left\|Q_{k}\right\|}} Q_{k}\right) z^{k} .
\end{aligned}
$$

The reason for this definition is that now

$$
\begin{aligned}
\Sigma\left(\bar{u}^{k}, \bar{y}^{k}\right) & =\int_{0}^{2 \pi}\left(\hat{y}_{\omega}^{k}-\hat{u}_{\omega}^{k}+\frac{1}{\sqrt{\left\|Q_{k}\right\|}} Q_{k} \hat{z}_{\omega}^{k}\right)\left(\hat{y}_{\omega}^{k}+\hat{u}_{\omega}^{k}+\sqrt{\left\|Q_{k}\right\|} \hat{z}_{\omega}^{k}\right)^{\mathrm{H}} d \omega \\
& =\Sigma\left(u^{k}, y^{k}\right)+Q_{k} \in Z .
\end{aligned}
$$

So we see that $\Sigma\left(\bar{u}^{k}, \bar{y}^{k}\right)$ is an element of $Z$ and, hence, $\bar{u}^{k}=\Delta^{k} \bar{y}^{k}$ for some contractive $\Delta^{k}$ of the form $(5,6)$. Finally consider

$$
\begin{aligned}
\left(I-\Delta^{k} M\right) \bar{u}^{k} \quad & \bar{u}^{k}-\Delta^{k} M\left(u^{k}+\left(\bar{u}^{k}-u^{k}\right)\right) \\
& =\bar{u}^{k}-\Delta^{k}\left(y^{k}+M\left(\bar{u}^{k}-u^{k}\right)\right) \\
=\bar{u}^{k}- & \Delta^{k}\left(\bar{y}^{k}+\left(y^{k}-\bar{y}^{k}\right)\right)-\Delta^{k} M\left(\bar{u}^{k}-u^{k}\right) \\
= & -\Delta^{k}\left(y^{k}-\bar{y}^{k}\right)-\Delta^{k} M\left(\bar{u}^{k}-u^{k}\right) .
\end{aligned}
$$

Using the fact that $\left\|\bar{u}^{k}-u^{k}\right\|_{2}=O\left(\sqrt{\left\|Q_{k}\right\|}\right),\left\|\bar{y}^{k}-y^{k}\right\|_{2}=O\left(\sqrt{\left\|Q_{k}\right\|}\right)$ and that $\lim _{k \rightarrow \infty}\left\|Q_{k}\right\|=$ 0 , we obtain from (18) that

$$
\lim _{k \rightarrow \infty}\left(I-\Delta^{k} M\right) \bar{u}^{k}=0, \quad \lim _{k \rightarrow \infty}\left\|\bar{u}^{k}\right\|_{2}=1 .
$$

This contradicts uniform robust stability.

Lemma 5.3. $\inf _{Z \in Z} \operatorname{Tr} E^{\mathrm{T}} Z$ is bounded from below for some $E \in \mathbb{R}^{n \times n}$ if and only if $E$ is of the form

$$
E=\operatorname{diag}\left(\tilde{E}_{1}, \ldots, \tilde{E}_{m_{r}}, E_{1}, \ldots, E_{m_{c}}, e_{1} I, \ldots, e_{m_{F}} I\right)
$$

with $\tilde{E}_{i}+\tilde{E}_{i}^{\mathrm{T}} \geq 0, E_{i}=E_{i}^{\mathrm{T}} \geq 0$ and $e_{i} \geq 0$.

Proof. Suppose that $\inf _{Z \in Z} \operatorname{Tr} E^{\mathrm{T}} Z$ is bounded from below. The off-diagonal blocks of $E$ are then zero for the following reason: Let $F$ be equal to $E$ but with its blocks on the diagonal equal to zero. The off-diagonal blocks of $Z \in Z$ are not restricted in any way so $Z:=\lambda F$ is an element of $Z$ for every $\lambda \in \mathbb{R}$. If $F$ is nonzero then $\operatorname{Tr} E^{\mathrm{T}} Z=\operatorname{Tr} E^{\mathrm{T}}(\lambda F)=$ $\lambda \operatorname{Tr} F^{\mathrm{T}} F$ and this is unbounded from below as a function of $\lambda$. Therefore $F$ must be zero, i.e., $E$ is block-diagonal.

The general form of a block-diagonal $E$ is

$$
E=\operatorname{diag}\left(\tilde{E}_{1}, \ldots, \tilde{E}_{m_{r}}, E_{1}, \ldots, E_{m_{c}}, \bar{E}_{1}, \ldots, \bar{E}_{m_{F}}\right)
$$

Express $Z$ as in (10). Then

$$
\operatorname{Tr} E^{\mathrm{T}} Z=\sum \operatorname{Tr} \tilde{E}_{i}^{\mathrm{T}} \tilde{Z}_{i}+\sum \operatorname{Tr} E_{i}^{\mathrm{T}} \bar{Z}_{i}+\sum \operatorname{Tr} \bar{E}_{i}^{\mathrm{T}} Z_{i} .
$$


Each block of $Z \in Z$ can vary independently of all other blocks of $Z$, so the only way that the above is bounded from below is that all

$$
\inf _{\tilde{Z}_{i}=\tilde{Z}_{i}^{\mathrm{T}} \geq 0} \operatorname{Tr} \tilde{E}_{i}^{\mathrm{T}} \tilde{Z}_{i}, \quad \inf _{\operatorname{He} \bar{Z}_{i} \geq 0} \operatorname{Tr} E_{i}^{\mathrm{T}} \bar{Z}_{i} \text { and } \inf _{\operatorname{Tr} Z_{i} \geq 0} \operatorname{Tr} \bar{E}_{i}^{\mathrm{T}} Z_{i}
$$

are bounded from below. It is fairly easy to show that

$$
\begin{aligned}
& \inf _{\tilde{Z}_{i}=\tilde{Z}_{i}^{\mathrm{T}} \geq 0} \operatorname{Tr} \tilde{E}_{i}^{\mathrm{T}} \tilde{Z}_{i}>-\infty \quad \Leftrightarrow \quad \mathrm{He} \tilde{E}_{i} \geq 0 \\
& \inf _{\mathrm{He}} \bar{Z}_{i} \geq 0 \operatorname{Tr} E_{i}^{\mathrm{T}} \bar{Z}_{i}>-\infty \quad \Leftrightarrow \quad E_{i}=E_{i}^{\mathrm{T}} \geq 0 \\
& \inf _{\operatorname{Tr} Z_{i} \geq 0} \operatorname{Tr} \bar{E}_{i}^{\mathrm{T}} Z_{i}>-\infty \quad \Leftrightarrow \quad \bar{E}_{i}=e_{i} I, 0<e_{i} \in \mathbb{R}
\end{aligned}
$$

(This is considered in more detail in [5].)

Acknowledgement: The authors would like to thank Fernando Paganini for his emails.

\section{References}

[1] M. Fan, A. Tits, and J. Doyle. Robustness in the presence of joint parametric uncertainty and unmodeled dynamics. IEEE Trans. on Aut. Control, 36(1):25-38, 1991.

[2] D. G. Luenberger. Optimization by Vector Space Methods. John Wiley, New York, 1969.

[3] A. Megretski. Necessary and sufficient conditions of stability: A multiloop generalization of the circle criterion. IEEE Trans. on Aut. Control, 38(5), 1993.

[4] A. Megretski and S. Treil. Power distribution inequalities in optimization and robustness of uncertain systems. J. Math. Syst. Estimation and Control, 3(3):301-319, 1993.

[5] G. Meinsma, Y. Shrivastava, and M. Fu. A dual formulation of mixed $\mu$ and on the losslessness of (D, G)-scaling. IEEE Trans. Aut. Control, 42(7):1032-1036, 1997.

[6] F. Paganini. Analysis of implicitly defined systems. In Proceedings of the 33rd CDC, pages 3673-3678, 1994.

[7] F. Paganini. Sets and Constraints in the Analysis of Uncertain Systems. PhD thesis, Caltech, USA, 1996.

[8] J. Shamma. Robust stability with time-varying structured uncertainty. IEEE Transactions on Automatic Control, 39(4):714-724, 1994. 